\title{
DIS2016 workshop summary
}

\section{Olaf Behnke*}

DESY, Notkestrasse 85, Hamburg 22607, Germany

E-mail: olaf.behnke@desy.de

\section{Markus Diehl*}

DESY, Notkestrasse 85, Hamburg 22607, Germany

E-mail: markus.diehl@desy.de

\section{Stefan Schmitt*}

DESY, Notkestrasse 85, Hamburg 22607, Germany

E-mail: stefan.schmitt@desy.de

\section{Matthew Wing*}

University College London, Currently at DESY, Notkestrasse 85, Hamburg 22607, Germany

E-mail: m.wing@ucl.ac.uk

We provide a brief summary of the 24th International Workshop on Deep Inelastic Scattering and Related Subjects (DIS2016), and also acknowledge sponsors and committee members.

XXIV International Workshop on Deep-Inelastic Scattering and Related Subjects

11-15 April, 2016

DESY Hamburg, Germany

${ }^{*}$ Editors, DIS2016 Proceedings. 


\section{Report}

This spring the 24th workshop on Deep Inelastic Scattering and Related Subjects (DIS2016) was held for the first time at DESY Hamburg, the place that with the first HERA ep data stimulated the foundation of the workshop series in 1993. Even now, two decades later, many new HERA physics results were presented at the workshop. However, perhaps more than ever before, DIS2016 acted as a melting pot of the worldwide investigations of the proton structure and strong interactions (QCD) at the LHC, HERA, TEVATRON, RHIC, JLAB, COMPASS and other experiments. It also covered the related theory advances and future experiments and provided a forum for high energy frontier results. This year's DIS workshop was attended by 327 participants representing 33 countries, presenting 270 talks on a variety of subjects. The workshop was organised in seven

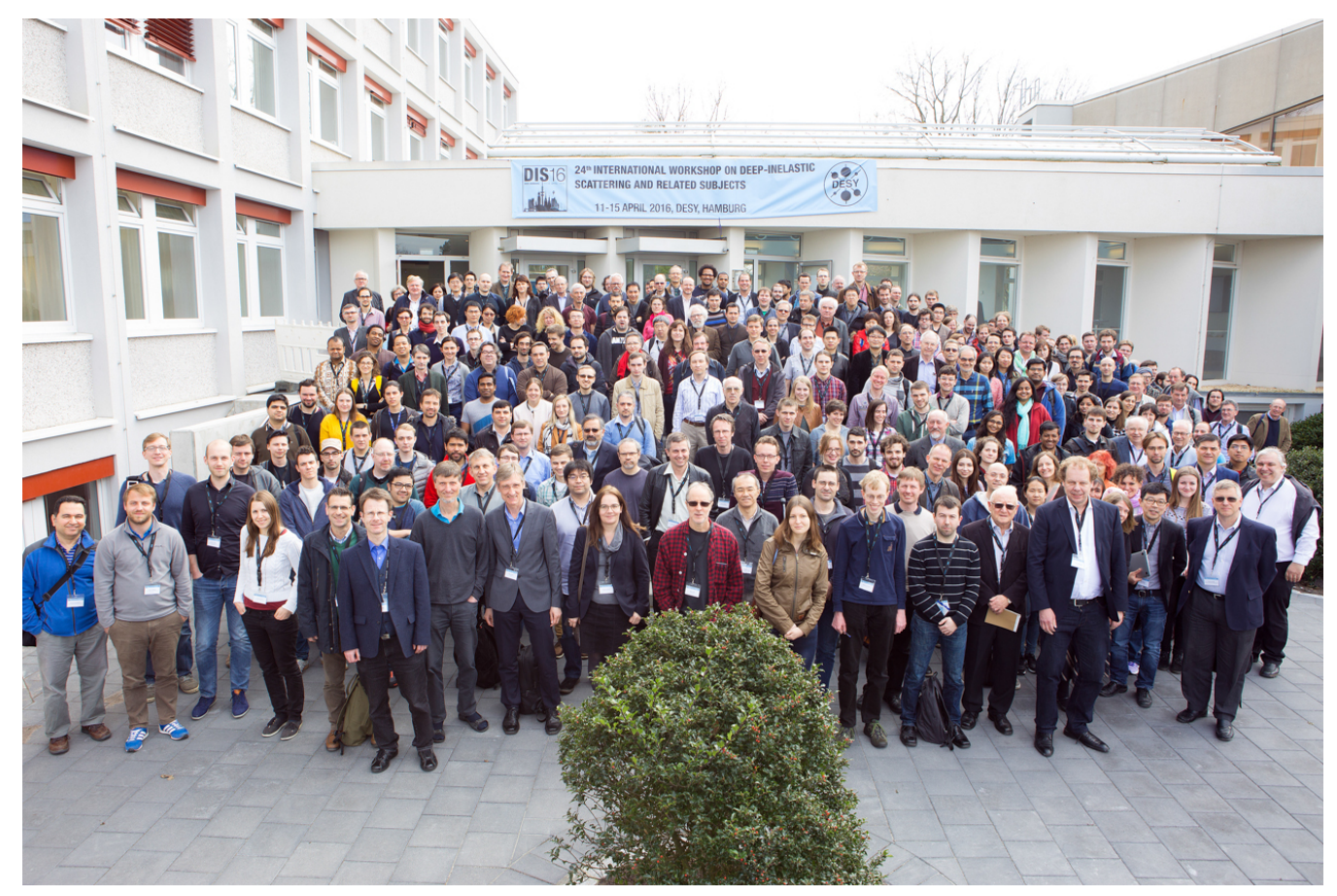

Figure 1: The DIS2016 workshop hosted 327 physicists from 33 countries.

separate working groups and after a full day of overview talks the groups met in parallel sessions and summarised their work in a final plenary session. In the following, a few selected highlights are given for each working group:

- Structure Functions and Parton Densities: The final H1+ZEUS combined inclusive DIS data, published in 2015, have been used by all main Parton Distribution Function (PDF) fitter groups and their significant impact on PDFs and resulting predictions of LHC processes was demonstrated at the workshop. PDF fits to these HERA data including higher twist effects help to diminish some tensions observed at low $\mathrm{x}$ values. ATLAS, CMS and LHCb presented the first LHC RUN II production measurements of $\mathrm{W}$ and $\mathrm{Z}$ bosons and of jets that will help to further constrain the PDFs. Alternative recommendations for best use and combinations of PDFs were presented and discussed. 
- Electroweak and BSM: Many final RUN I results were reported by ATLAS and CMS on the $125 \mathrm{GeV}$ Higgs like particle as well as on precision measurements of multiboson production (WW, Z $\gamma \gamma$, etc.) probing anomalous couplings. No deviations from the SM were observed. The need was pointed out to complement the increasing precision of electroweak LHC measurements by calculations including Next-to-Leading Order (NLO) electroweak corrections and ongoing generator implementation work was presented. The ZEUS experiment showed the first observation of a running effective $\sin ^{2} \theta_{W}$ obtained from a single machine.

- QCD and Hadronic Final States: A wealth of new LHC results was presented on the production of jets as well as photons, $\mathrm{W}$ and $\mathrm{Z}$ bosons alone or together with jets, and also showing first RUN II results. These studies often probe the final state kinematics in great detail. The theorists try to match the improved experimental precisions e.g. with generators based on full Next-to-Next-to-Leading Order (NNLO) QCD Matrix elements plus parton showers which are being established now for $2 \rightarrow 1$ processes. The description of the underlying event and double-parton scattering was another important topic, with many new experimental and theoretical results being debated.

- Heavy Flavours: Brand new CMS top-pair production data from RUN II were compared to the first complete NNLO QCD predictions for differential distributions that became available shortly before the workshop. These calculations improve the description of the observed top quark transverse momentum spectrum and in general open a new precision era in top physics. Many new beauty and charm results were presented such as the first RUN II measurements of open charm production by LHCb exploiting their new quasi-online measurement scheme.

- Low x, Diffraction and Vector Mesons: Theorists propose to do extended studies at LHC with events containing Mueller-Navelet jets, i.e. two forward jets with a large rapidity gap between them. This topology is particularly sensitive to BFKL QCD dynamics, characterized by unordered gluon radiation which should be reflected by angular and momentum distributions of multi-jets produced in the events. Several theory advances were presented on the Colour Glass Condensate and Colour Dipole model phenomenology of diffractive vector meson production at HERA, where also the first measurement of the reaction $\gamma+p \rightarrow \rho^{0} \pi^{+} n$ was reported by H1. Ultra-Peripheral Collisions in nuclear collisions at LHC and RHIC are another hot topic and the ALICE results for $\mathrm{PbPb} \rightarrow \mathrm{PbPb}+\mathrm{J} / \psi$ indicate a moderate gluon shadowing in the lead nucleus.

- Spin Physics: Concerning the long-standing spin puzzle - the question how the nucleon's spin is distributed amongst its constituent partons - new spin asymmetry results were presented by COMPASS as well as by PHENIX and STAR, adding significant constraints on the quark and gluon spin contributions. New Deeply Virtual Compton Scattering $\gamma^{*} p \rightarrow \gamma p$ measurements were shown by COMPASS and JLAB Hall-A/B, to be used for improving the 3-D Generalized Parton Distributions functions, which may be related to the quark and gluon orbital momentum. COMPASS, HERMES and JLAB presented new studies of semiinclusive DIS, where, in addition to the outgoing electron, at least one hadron is detected whose kinematics provides information on the transverse-momentum-dependent parton dis- 
tributions. Further clues on that are provided by the first measurement of the transverse single-spin asymmetry of Drell-Yan weak boson production performed by STAR.

- Future experiments: JLAB will continue the 3-D hadron imaging studies with the second generation of experiments using its $12 \mathrm{GeV}$ electron beam while COMPASS and STAR will take further data until 2017. Despite the achievements by these facilities the lack of experimental information for $x \leq 0.01$ will remain a severe limitation. There is great hope that the Electron Ion Collider (EIC) project in the US will be the future machine that will lead - with centre-of-mass energies not too far below HERA - to a comprehensive 3-D hadron imaging and solve the spin puzzle. After the EIC received in the 2015 long range planning of the NSAC the "highest priority recommendation for new construction", preparatory studies at JLAB and BNL, each of them proposing to host the collider, are in full swing. For both proposals important updates were presented including further physics prospects. On the energy frontier the intriguing opportunity of an electron ion collider (with $\sqrt{s}$ up to $\sim 4 \mathrm{TeV}$ ) in the envisaged $54 \mathrm{~km}$ ring accelerator complex in China was reported, competing with the LHeC and FCC-he proposals at CERN. Such machines could provide a unique precision tool for a complete flavour decomposition of the proton structure for $\mathrm{x}$ values from $10^{-7}$ to 1 and would cover a broad spectrum of strong and electroweak physics.

On the closing day the first Guido Altarelli prize was awarded to the two outstanding young researchers Fabrizio Caola and Jan Kretzschmar (see CERN Courier June 2016) and the scientific program was concluded with a talk on the future perspectives by current ECFA chair Halina Abramowicz.

Several nice social events rounded off the workshop: a reception in the majestic Hamburg town hall, Brian Foster's public lecture on "Einstein and Music" followed by a performance of the "Schubert Octet Concert", a conference dinner in the restaurant "Schulauer Fährhaus" on the banks of the river Elbe and excursions to interesting sights in Hamburg such as the huge Airbus production plant.

In summary, the DIS2016 workshop once again provided a comprehensive overview of the worldwide research on proton structure, QCD and physics at the energy frontier. It will be very interesting to follow the future developments - an ideal opportunity for this is the next DIS workshop which will take place in Birmingham, UK, from 3 to 7 April 2017.

\section{Acknowledgements}

The workshop was jointly organised by DESY and the University of Hamburg. The workshop was generously supported by Deutsche Forschungsgemeinschaft (DFG), CERN, Brookhaven National Laboratory (BNL), Jefferson Lab and DESY.

As editors, we would like to thank the members of the International Advisory Committee for allowing DESY to host this meeting and the Local Organizing Committee for selecting outstanding speakers and conveners and for a flawless execution of the local logistics. We thank the DESY directorate for their strong support and encouragement. Finally we would like to thank the numerous local helpers at DESY.

The DIS2016 conference website is: https://dis2016.desy.de 


\section{International Advisory Committee (IAC)}

Halina Abramowicz (Tel Aviv)

Barbara Badelek (Warsaw)

Sergio Bertolucci (CERN)

Ian Brock (Bonn)

Allen Caldwell (Munich)

Amanda Cooper-Sarkar (Oxford)

John Dainton (Liverpool)

Cristinel Diaconu (Marseille)

Rolf Ent (JLAB)

Joel Feltesse (Saclay)

Stefano Forte (Milano)

Elisabetta Gallo (DESY)

Rolf-Dieter Heuer (CERN)
Max Klein (U Liverpool)

Aharon Levy (Tel Aviv, Chair)

Lev Lipatov (St. Petersburg)

Joachim Mnich (DESY)

Berndt Mueller (BNL)

Hugh Montgomery (JLAB)

Rosario Nania (Bologna)

Fredrick Olness (SMU Dallas)

Juan Terron (Madrid)

Robert Thorne (London)

Katsuo Tokushuku (KEK)

Matthew Wing (DESY / London)

\section{Local Organizing Committee (LOC)}

Olaf Behnke (Chair)

Markus Diehl

Inna Henning

Roman Kogler

Katja Krüger
Jenny List

Armine Rostomyan

Stefan Schmitt

Thomas Schörner-Sadenius

Matthew Wing 